\title{
Sartre par Barbaras : mouvements d'une lecture critique
}

Fernanda Alt

\section{(2) OpenEdition}

1 Journals

Édition électronique

URL : https://journals.openedition.org/alter/2239

DOI : $10.4000 /$ alter.2239

ISSN : 2558-7927

Éditeur :

Association ALTER, Archives Husserl (CNRS-UMR 8547)

\section{Édition imprimée}

Date de publication : 1 novembre 2020

Pagination : $353-370$

ISBN : 978-2-9550449-6-4

ISSN : $1249-8947$

\section{Référence électronique}

Fernanda Alt, "Sartre par Barbaras : mouvements d'une lecture critique », Alter [En ligne], 28 | 2020, mis en ligne le 22 décembre 2020, consulté le 12 décembre 2021. URL : http://

journals.openedition.org/alter/2239; DOI : https://doi.org/10.4000/alter.2239

Ce document a été généré automatiquement le 12 décembre 2021.

Revue Alter 


\title{
Sartre par Barbaras : mouvements d'une lecture critique
}

\author{
Fernanda Alt
}

Il n'y a pas d'autre manière de penser le mouvement en sa vérité que de l'effectuer

R. Barbaras, De l'être du phénomène

D'une manière générale, il est vrai que Renaud Barbaras est critique de Sartre, au sens où il souligne avant tout les problèmes, les échecs et les impasses de sa philosophie. De plus, Barbaras étant une référence dans les études merleau-pontyennes, outre la reconnaissance de la portée de sa propre philosophie, il est naturel d'attribuer une certaine continuité entre les positions de Merleau-Ponty et les siennes par rapport à Sartre. Et ceci pour deux raisons : d'une part, Barbaras reprend cette critique dans une écriture qui va au-delà du commentaire, en la radicalisant ou la potentialisant ; d'autre part, au cours du développement de sa philosophie, il s'oppose aux problèmes sartriens déjà soulevés par Merleau-Ponty, même si c'est pour les exprimer autrement, pour trouver de nouvelles solutions, par divers chemins. Ainsi, ce n'est pas seulement en tant que prolongement et potentialisation de la critique de Merleau-Ponty que l'on peut entendre la lecture de Sartre par Barbaras. En effet, on y trouve des mouvements qui vont au-delà des points précédemment posés; des mouvements qui ne se limitent pas à un adressage frontal d'opposition et de réfutation, car ils révèlent des nuances qui méritent d'être soulignées si l'on veut comprendre la relation entre les auteurs, ainsi que les tensions qui se sont développées au sein de la philosophie de Sartre. En ce sens, plutôt que de fournir une présentation exhaustive de la critique de Barbaras à Sartre, voire une sorte de mise à l'épreuve de la validité, des contradictions, etc. de cette critique, nous souhaitons avant tout montrer la dynamique d'une relation faite de rapprochements, de distances, de condamnations, de possibilités ouvertes, entre autres, qui caractérise un mouvement vivant qui est la reprise d'un auteur comme expérience de la pensée. 


\section{La critique de Merleau-Ponty potentialisée : le dualisme fondamental de la philosophie sartrienne.}

2 Pour ceux qui cherchent à comprendre les éléments en jeu dans la critique de Sartre par Merleau-Ponty dans Le visible et l'invisible, le chapitre trois de la deuxième partie "Être et Néant: la dialectique »- de De l'être du phénomène de Barbaras est éclairant, mais il ne faut pas en conclure qu'il s'agit simplement d'un commentaire. Dans celui-ci, le mouvement de l'auteur finit par radicaliser et potentialiser la critique merleaupontyenne. Le point de convergence avec Merleau-Ponty est donc très évident: Barbaras reconnaît que Sartre n'arrive pas à surmonter les impasses et les limites des philosophies réflexives et que sa conception du sujet pour-soi comme néant est problématique. Il radicalise la position merleau-pontyenne à sa manière, c'est-à-dire l'idée que la philosophie du négatif de Sartre aboutit à une philosophie du survol, conduisant à la conception d'un sujet qui finalement n'appartient pas au monde. Mais la façon dont l'auteur nous amène à de telles conclusions est tout à fait singulière, puisqu'elle montre que Sartre s'approche de Merleau-Ponty dans un mouvement d'opposition à Husserl, mais pour offrir une solution différente. Pour Barbaras, au lieu de penser une unité entre l'être et le néant dans l'expérience d'ouverture au monde, comme le fait Merleau-Ponty, Sartre part d'une opposition radicale entre les termes qui renouvelle le dualisme en même temps qu'il le dépasse. Si Sartre ne peut pas concevoir un mélange efficace de ce qui était précédemment opposé, la relation entre le pour-soi et l'en-soi ne peut être envisagée que sur un plan logique, et non comme une expérience concrète. Comme le dit Barbaras, en accord avec Merleau-Ponty :

En réalité cette philosophie ne nous livre que «le portrait abstrait d'une expérience $»^{1}$ : elle incorpore au pour-soi et à l'en soi le passage de l'un à l'autre que l'expérience atteste, mais le mouvement n'est pas restitué. Le passage ne saurait être effectif dès lors que, sous les termes d'Être et de néant, la fixité des catégories réflexives est maintenue: il ne s'accomplit alors qu'au prix de renouveler leur opposition ${ }^{2}$.

Mais quels sont les enjeux des "philosophies réflexives", pour que le chemin permettant de les dépasser devienne un piège pour Sartre? Merleau-Ponty montre que ce dernier s'oppose au cadre réflexif qui présupposait une positivité intérieure et traitait l'extérieur comme simple négatif, proposant un esprit comme pur négatif et donc l'être comme extérieur et comme pure positivité. Ainsi, une simple inversion entre les sphères positive et négative ne surmonte pas le dualisme, de la même manière que Heidegger disait que «le renversement d'une proposition métaphysique reste une proposition métaphysique $»^{3}$. Au sujet des philosophies réflexives, Barbaras souligne le rejet merleau-pontyen d'une division que cet auteur identifie dans la philosophie de Descartes entre un plan irréfléchi de la vie et un plan scientifique réflexif, ainsi que la scission husserlienne entre le plan de l'attitude naturelle et celui révélé par l'attitude transcendantale. Merleau-Ponty cherche une unité qui nous permette de penser l'ouverture sur le monde, de telle sorte que

[...] loin de se confondre avec un contact aveugle l'en soi, elle apparaisse comme le commencement de la réflexion [...] Cela signifie qu'inversement, il faut définir la réflexion, non pas comme procédant d'une sphère positive et autonome du point de vue de laquelle l'ordre de l'irréfléchi serait définitivement incompréhensible, mais comme ce qui tire sa possibilité la plus propre de l'irréfléchi lui-même. Bref, il s'agit de montrer comment «l'entrer en soi » est identiquement « sortir de soi »". 
Ainsi établi, ce dualisme fait en sorte que l'inversion qui donne la primauté à l'irréfléchi chez Sartre conduit avec elle au maintien de la division des plans, même si elle vide le sujet de toute positivité, le caractérisant comme pur néant. C'est ici que Barbaras montre comment Merleau-Ponty et Sartre, après avoir brièvement convergé sur l'identification d'un problème, suivent des orientations différentes et décisives dans leur philosophie. Tous deux critiquent une sorte de "substantialisation" de la subjectivité husserlienne, qui empêche le mouvement même de la conscience intentionnelle. Dans La Perception, ce point est clarifié à partir de la question suivante : "Comment un vécu immanent serait en mesure de faire apparaître une transcendance, c'est-à-dire finalement de faire sortir la conscience d'elle-même. N'y a-t-il pas une difficulté à penser comme contenu de conscience l'acte sur lequel repose le rapport de la conscience à son autre ? $»^{5}$. Sartre, quant à lui, a posé une question assez similaire dans l'«Introduction » de L'Être et le Néant en disant que Husserl préfère réduire l'être au " non-être ", en faire un noème « irréel », instaurant finalement la réalité $\left(\right.$ réel $\left.^{6}\right)$ dans la plénitude subjective. En ce sens, Sartre affirme que cette subjectivité ne « saurait sortir de soi pour poser un objet transcendant » alors que la réalité de l'objet est basée sur la plénitude subjective et que son objectivité est un non-être, de sorte que «jamais l'objectif ne sortira du subjectif, ni le transcendant de l'immanence, ni l'être du nonêtre $»^{7}$. Le point de proximité que nous identifions dans ces questions correspond à la critique d'un immanentisme de la subjectivité husserlienne qui finit par admettre des " contenus », bien que Sartre l'oriente ensuite vers le noema tandis que, pour Barbaras, elle se limite au vécu lui-même. Ce dernier pense qu'Husserl fait du vécu un être positif et ne respecte pas l'évanescence de l'apparaître, ce qui l'amène d'une certaine manière à intérioriser l'empirisme et l'intellectualisme au "sein de la conscience transcendantale sous la forme de la dualité et de l'unité finalement incompréhensible de la matière et de la forme $»^{8}$. Toutefois, Barbaras considère que la théorie des esquisses de Husserl, contrairement à la critique de Sartre, est justement celle qui légitime la transcendance : "Si tant est qu'une chose (un "quelque chose") est bien une réalité transcendante, autrement dit se distingue de mes vécus, elle ne sera présente comme chose qu'à la condition de ne pas l'être tout entière, de différer une donation adéquate, de résister à l'appropriation ${ }^{9}$. Sartre ne voit pas de la même façon cet aspect souligné par Barbaras sur la théorie des esquisses de Husserl. Alors que Sartre croit que la subjectivité husserlienne, tout comme l'« Esprit-araignée » - propre aux philosophies idéalistes qui transposent toute réalité transcendante à l'intérieur de celui-ci ${ }^{10}$ - admet des contenus dans la subjectivité pure, transformant l'être perçu en son corrélat, Barbaras insiste sur le fait que ce sont précisément les donations par esquisses qui font que la chose perçue échappe à la connaissance que l'on peut en avoir, du fait qu'elle "excède toute expérience qui peut en être faite, et cet excès est sans mesure, l'écart entre la chose et sa perception singulière ne saurait être réduit " ${ }^{11}$. Le point que Barbaras considère extrêmement important dans la théorie de Husserl n'est pas compris de cette manière par Sartre, puisque ce dernier dénonce le fait que les apparences sont réduites à une "matière impressionnnelle subjective $»^{12}$, de sorte que si Husserl définit la conscience comme transcendance et fait de l'être "un être perçu ", il est « totalement infidèle à son principe $»^{13}$. Ainsi, dans la mesure où Sartre ne voit pas la théorie des esquisses comme un moyen de contester l'idéalisme et de légitimer la sphère transcendante, il prétend éviter ces problèmes par la "transphénoménalité " des modes d'être du pour-soi et de l'en-soi. Le pour-soi est alors engagé dans la transcendance en ce sens qu'il échappe à une philosophie contemplative husserlienne, 
qui présuppose une conception de la conscience comme une sphère hypostasiée de la subjectivité. Sartre nomme " preuve ontologique » cet engagement qui consiste en une version particulière du principe de l'intentionnalité de la conscience : «La conscience est conscience de quelque chose: cela signifie que la transcendance est structure constitutive de la conscience ; c'est-à-dire que la conscience naît portée sur un être qui n'est pas elle ${ }^{14}$. Ce qui signifie, dit Sartre, que la conscience est "intuition révélante » de quelque chose qu'elle n'est pas, de sorte que cette intuition n'est pas un rapport de connaissance, mais d'être. La conscience n'existe qu'en tant que révélation de quelque chose, impliquant «dans son être un être non conscient et transphénoménal »" n'existe que par cette intuition révélante de l'être, la négation interne, de sorte qu'il n'est pas possible de concevoir une subjectivité en dehors de cette relation négative. D'autre part, l'être à partir duquel la conscience existe comme « intuition révélante » est l'être transphénoménal de phénomènes qui, ne pouvant pas être immanent à la conscience, doit être lui-même en-soi. Sur ce point, Sartre s'éloigne des positions fondamentales de Husserl et est même son contrepoint, souligne Barbaras, car il vide la conscience et restitue à l'objet sa réalité : « Ainsi, Sartre oppose le vide de la conscience à la plénitude impressionnelle des vécus, et la densité de l'en-soi au non-être de l'objet. Il attribue à la conscience le manque par lequel Husserl définissait l'objet et à l'en-soi la plénitude par laquelle Husserl caractérisait le subjectif $»^{16}$. Voici une autre façon de décrire cette «inversion» sartrienne. Parmi les conséquences significatives de ce geste, on peut signaler une rechute de Sartre tant dans le réalisme que dans l'idéalisme, une dichotomie qu'il a prétendu avoir dépassée dans l'«Introduction » de L'Être et le Néant. La voie réaliste est due à la conception matérielle et positive de l'en-soi, étant donné que le refus de l'idéalisme conduit ici à l'effort de ne jamais réduire l'être à son apparition pour la conscience, de sorte que l'être des phénomènes - l'être en-soi - est qualifié de «transphénoménal ». Or, si l'en-soi échappe d'une manière ou d'une autre au phénoménal, on peut se demander si cette ontologie peut maintenir son statut phénoménologique, car elle reconnaît un certain réalisme non phénoménal. Ce point est soulevé par Barbaras dans le texte "Désir et manque dans L'Être et le Néant : le désir manqué », où il remet en cause ce statut. Dans la mesure où Sartre " pose l'en-soi hors de sa phénoménalité et postule donc une sorte d'indépendance de l'en-soi vis-à-vis du pour-soi $[. ..] »^{17}$, il en résulte " une soumission à l'attitude naturelle, compromettant gravement la démarche phénoménologique ${ }^{18}$. Enfin, il s'agit d'une " phénoménologie sans phénomènes ", puisque :

En parlant d'en-soi, Sartre introduit donc d'emblée, sur un mode finalement réaliste, une scission, entre l'être et le phénomène. Au lieu de partir de la phénoménalité comme l'élément au sein duquel le sens d'être du sujet et du transcendant (en-soi) peuvent être conquis, il reconstitue au contraire la phénoménalité à partir de la relation entre le pour-soi et un en-soi reposant en luimême ${ }^{19}$.

5 La conception de l'en-soi comme pure positivité est donc un contrepoint symétrique à cette subjectivité vidée au point d'être caractérisée comme pur néant. Nous retrouvons ici l'autre conséquence du geste sartrien d'inversion mentionné ci-dessus. Barbaras adopte la position de Merleau-Ponty dans Le Visible et l'Invisible, qui considère que le problème réside dans la conception sartrienne de la négativité. Alors que MerleauPonty s'oppose à la positivité de l'essence husserlienne en la "décomprimant", cherchant à revenir au point d'insertion de la subjectivité dans l'être, Sartre se prive de la possibilité de penser effectivement le négatif en concevant une négativité positive, aussi contradictoire qu'une telle expression puisse résonner ${ }^{20}$. Pour Merleau-Ponty - de 
même que pour Barbaras - cette négativité est le néant sartrien. La différence entre négativité et néantité devient alors fondamentale pour Barbaras, car c'est en raison de cette différence qu'il développe sa conception du sujet comme mouvement, comme en témoigne l'œuvre Dynamique de la manifestation: "Le mode d'exister que désigne la négativité en sa différence avec le néant doit donc être défini comme mouvement ${ }^{21}$. Selon Barbaras, le problème ne consiste donc pas à penser le sujet comme négativité, étant donné que c'est par la négativité que se fait la différence ontologique entre cette manière d'être et les autres étants intramondains - ici la différence comme mouvement -, mais plutôt à penser la négativité comme néantité et donc l'étant comme en-soi :

D'une part, ce passage du non-étant au néant, ou encore la subordination de la négativité à la néantité a pour présupposé une détermination pauvre et, pour ainsi dire, abstraite, de l'étant. C'est en effet parce que l'étant est compris comme une entité absolument dense, identique à elle-même et donc immuable, comme une sorte d'hyper-objet, à la manière de l'Être parménidien, que sa négation ne peut renvoyer alors qu'à un néant absolument extérieur, à un pur néant qui est aussi négatif que l'être était positif, qui est aussi ontologiquement inconsistant que l'être était ontologiquement dense ${ }^{22}$.

6 C'est pour cela que Merleau-Ponty parle de parallélisme entre l'être et le néant, car si le néant est bien une sorte de chose, à cause de sa réification, les deux termes sont finalement positifs, de sorte que leur relation est parallèle, frontale et sans mouvement ${ }^{23}$. Barbaras suit cette critique dans ses résultats, en particulier concernant le fait qu'un tel parallélisme empêche le mouvement propre à une dialectique. En ses termes, il qualifie donc la philosophie du négatif de Sartre comme une philosophie de l'identité, qui n'admet aucune différence. Même si chez Sartre un terme ne se définit que par rapport à l'autre, cette relation est abstraite et ne les modifie pas. Au contraire, elle renforce l'identité de chacun dans la mesure où le néant n'est un pur néant que si l'être est un pur être :

En définissant la conscience comme néant, Sartre ne montre pas comment elle ouvre effectivement à l'Être, il se contente de la déterminer de telle sorte qu'elle ne peut être pensée sans recours à L'Être : il met en évidence une nécessité pour la pensée, une nécessité abstraite, mais n'accompagne pas le mouvement effectif. Il s'agit bien de conférer à chaque terme une détermination telle que son identité ne puisse se soutenir que de la position de l'autre terme. Penser selon le négatif pur, c'est déjà s'être donné l'identité ou la positivité ; penser selon la pure densité de l'Être, c'est déjà avoir posé la pure négativité du néant $[. . .]^{24}$.

7 Si la relation est donc l'opérateur qui renforce l'identité de chaque terme avec luimême, il n'y a pas de conditions pour qu'il y ait de la différence effective. C'est-à-dire que nous restons sur un plan d'opposition logique mené par la pensée qui n'atteint pas le mouvement concret qui se produit dans l'expérience d'ouverture au monde: «l'ouverture au monde est schématisée sous la forme d'une nécessité d'entendement, qui procède de la définition préalable des termes par leur opposition ${ }^{25}$. En réalité, alors que Merleau-Ponty parle de « repos » des termes opposés, Barbaras évoque plutôt un mouvement caricatural, un va-et-vient schématique, ce qui fait que Sartre renouvelle le dualisme en même temps qu'il cherche à le surmonter.

De ce fait, Barbaras assume et développe la critique de Merleau-Ponty de manière à la potentialiser, en mettant en évidence les mouvements d'inversion des dualismes menés par Sartre qui ne le libèrent pas d'une philosophie dualiste, mais qui témoignent au contraire de sa revitalisation. Ce geste sartrien entraîne des conséquences fondamentales sur sa philosophie et sur certains thèmes repris de façon originale par 
Barbaras, à savoir la question de l'autre et la notion de désir. L'auteur se consacre à présenter ces conséquences à travers un mouvement qui révèle à la fois proximités et distances, mouvement fondamental pour le développement de sa propre philosophie.

\section{La question d'autrui}

9 Merleau-Ponty montrait déjà comment le dualisme fondamental de la philosophie sartrienne empêchait l'expérience effective de l'altérité. Cela est dû au fait que la division que Sartre établit entre les manières d'être pour-soi et pour-autrui résulterait de la conception du sujet comme une pure sphère de néantisation, qui n'acquiert son objectivité que par le regard de l'autre, radicalisant la séparation entre les sujets et ratifiant finalement le caractère solipsiste de cette philosophie. Ainsi, au lieu d'attester d'une "rencontre" avec l'autre, ou d'une subjectivité dont l'altérité fait partie intégrante, Sartre, par sa théorie du pour-autrui, finit par isoler davantage le sujet dans une sphère «intouchable». Barbaras explore cette conséquence en montrant que «la relation à autrui se confond avec l'expérience du pour-autrui : la conscience n'a jamais affaire à l'autre en personne, mais seulement à elle même » et que "si l'accès à autrui se confond avec l'épreuve de mon être-regardé, il cesse d'être une expérience, faute de révéler quelque chose dont il soit l'expérience : il revient à la découverte de mon êtrepour-autrui, c'est-à-dire d'une structure de ma conscience ${ }^{26}$ de sorte que, comme le concluait déjà Merleau-Ponty, « je n'ai pas affaire aux autres, j'ai affaire tout au plus à un non-moi neutre, à une négation diffuse de mon néant $"^{27}$. À la suite de MerleauPonty, Barbaras montre également combien cette théorie de l'autre est imprégnée des présupposés d'une "philosophie de la vision", au sens d'une philosophie d'un sujet non incarné, qui survole le monde au point de le prendre en panorama devant lui, tel un néant pur face à l'être, ignorant l'essence même du « voir ». Envisager l'expérience de l'autre à travers le regard révèle donc la cohérence du déroulement, sur cet autre plan, de la philosophie de la vision, dans la mesure où Sartre s'attache à un type d'expérience, celle du regard: "Philosophie de la vision, la pensée du néant s'accomplit, au plan de l'expérience d'autrui, comme philosophie du regard $»^{28}$, conclut Barbaras.

Outre cette reprise de la critique de Merleau-Ponty, soulignons la manière dont Barbaras développe d'autres conséquences de la théorie de l'autre chez Sartre. Le texte «Le corps et la chair » dans la troisième partie de L'Être et le Néant reprend ces points en les approfondissant, et va encore plus loin en mettant en lumière de façon novatrice et inattendue un aspect intéressant de la pensée sartrienne, à savoir le concept de chair. Avant d'aborder ce point, Barbaras développe une longue et rigoureuse discussion sur la théorie sartrienne de l'autre sur laquelle nous n'entrerons pas dans les détails ici $^{29}$. L'auteur y souligne certains aspects, à commencer par les conditions établies par Sartre sur sa théorie de l'autre : 1) la relation à l'autre est interne et non externe (comme dans le cas de la connaissance), c'est une relation d'être ; 2) le seul point de départ possible est l'intériorité du cogito ; 3) la relation à l'autre ne peut être de constitution ; il ne peut pas être déduit d'une structure ontologique. Nous ne pouvons pas détailler tous ces points, mais nous mettons ici en évidence le fait que Barbaras trouve une contradiction dans les conditions établies par Sartre pour ensuite proposer une solution qui reste sartrienne, bien qu'elle soit une solution dont Sartre lui-même ne puisse tirer parti, du fait de sa fidélité au dualisme initial. Barbaras montre d'abord comment ce dualisme 
guide les analyses sartriennes du corps, dans la mesure où elles dépendent de la théorie de l'autre :

L'analyse de Sartre repose donc tout entière sur l'opposition tranchée entre une conscience irréfléchie qui est définie par l'immanence pure et une objectité qui ne saurait appartenir au pour-soi comme tel, qui relève du connu et, partant, renvoie à autrui. Le pour-soi n'a pas de corps et, loin que son être pour-autrui procède de son appartenance à l'extériorité, c'est cette appartenance qui repose tout entière sur autrui. Le pour-soi s'incarne, mais ce n'est pas en tant que pour-soi qu'il s'incarne; s'il est capable de passer dans l'extériorité, ce passage n'appartient cependant pas à l'essence de la présence à soi ${ }^{30}$.

Barbaras comprend alors que l'incarnation du pour-soi - son inscription dans l'extériorité - n'est possible que par la voie de l'être-pour-autrui. Pour cette raison, le fait que Sartre conçoive un corps pour-soi, un corps vécu, ne garantit pas qu'une telle incarnation se produise dans la dimension pour-soi, qui est la sphère immanente purifiée: «en tant que néantisation ou translucidité, le pour-soi ne peut qu'être étranger à toute extériorité $~_{31}$, conclut-il. La preuve de l'absence d'incarnation dans la sphère du pour soi-même est attestée par la posture de Sartre par rapport aux expériences propres au corps vécu, comme, par exemple, son refus de la sensation d'effort de Maine de Biran, mentionnée brièvement dans L'Être et le Néant. En outre, même si Sartre ne le contourne pas, il inessentialise le phénomène de la double sensation déjà mis en évidence par Husserl dans les Ideen II $^{32}$. Phénomène qui atteste précisément de l'incarnation du corps propre en rendant sensible le corps objectivé, privilégiant le toucher par rapport à la vision ; une expérience où « [par le toucher] une extériorité spatiale advient comme mienne, un sentir déploie son propre espace, s'incarne $»^{33}$. Du refus de l'originalité du phénomène du toucher, Sartre pousse alors la philosophie de la vision à son extrême, car outre le fait que ce modèle présuppose un néant désincarné face au monde, il fait de l'incarnation un phénomène procédant du regard de l'autre, un autre lui aussi transmondain, non situé.

Ce qui est intéressant, c'est que les analyses de Barbaras proposent un argument qui conteste le dualisme même qui soutient sa critique: après avoir mentionné le "silence $»^{34}$ sartrien sur le corps pour-soi, l'auteur se tourne vers la facticité qui témoigne de l'engagement du pour-soi dans le monde, d'un corps qui fait de lui un être situé, qui n'est pas objet. Il y mentionne la présence d'une affectivité originelle et l'idée de chair chez Sartre comme une contestation des conditions préalablement établies. Par l'affectivité originelle, Sartre montre que le pour-soi a une appréhension immédiate de son corps, une sorte de goût de soi (terme de Sartre) qui instaurerait une passivité ou un centre d'opacité à ce qui était jusqu'alors considéré comme pure translucidité. Par l'idée de chair, il est possible d'appréhender la facticité du corps de l'autre directement par le niveau factice, qui échappe à l'objectivation. Ainsi, le corps d'autrui se présente comme un corps vivant, "psychisme incarné » dans le monde, garantissant aussi l'expérience de l'altérité en dehors des modèles d'objectivation de l'être-pour-autrui. Ces surprises sartriennes, après tous les efforts déployés pour assurer l'expérience de l'autre par les conditions évoquées ci-dessus, laissent Barbaras «perplexe $»^{35}$. Il se demande par exemple si l'idée d'affectivité originelle ne parviendrait pas à "brouiller la translucidité ${ }^{36}$ de la conscience et ne rendrait finalement pas inutile voire inadaptée l'analyse du pour-autrui qui se fonde sur l'expérience du regard. Toutefois, il considère que l'« hypercartésianisme » de Sartre 
est si fort qu'il ne permet pas, même avec l'émergence de ces nouvelles forces, l'implosion du dualisme de base.

13 C'est ce dualisme qui fait que Sartre se concentre sur l'analyse du pour-autrui et de l'expérience de l'altérité par la négation interne: l'appréhension de l'autre passe par mon être-regardé dans l'expérience de la honte, qui confère une extériorité au poursoi. Le dualisme qui s'y instaure se situe donc entre la sphère pour-soi subjective et la sphère pour-autrui objective, médiatisée et rendue possible par le regard de l'autre. Cependant, la troisième condition ci-dessus montre la fragilité de toute cette théorie : l'apparition de l'autre est une "rencontre ", aussi parce qu'il ne peut être déduit du pour-soi. Ainsi, l'incarnation du pour-soi dépend d'un événement contingent, qui à son tour a le rôle de fondement de l'expérience de la négation interne, c'est-à-dire de la reconnaissance dans l'intériorité d'un être-pour-autrui. Une rencontre qui expose au risque l'expérience de l'altérité d'une manière générale : s'il peut y avoir une certitude dans le cogito, cette certitude est tributaire d'un «événement ». De plus, dans la mesure où nous restons dans une appréhension centrée sur le cogito, il n'y a pas d'accès effectif à l'autre, mais seulement à une dimension étrangère de soi. Cela dit, les notions d'affectivité originelle et de chair ébranleraient alors les conditions mêmes établies par Sartre en vue de rendre la démarche argumentative sur l'être-pour-autrui insuffisante pour la compréhension de la question de l'autre. En ce sens, Barbaras a raison de montrer combien Sartre a tout misé sur l'expérience du regard au point de ne pas suffisamment développer les conséquences de cet accès factice non objectivant attesté par la chair dans sa philosophie.

14 Toutefois, cette voie tracée par Barbaras, plutôt que d'attester un autre échec de Sartre concernant son dualisme, vient affaiblir celui-ci face aux preuves des différents niveaux de réalisation de la facticité compris dans l'expérience de l'autre. Pour cela, il faut distinguer la facticité de l'objectivité, traitées en termes d'engagement et d'enracinement par Sartre: si le pour-soi est engagé dans le monde (inscrit par la structure immédiate de la facticité), le pour-autrui, en tant qu'objectivation de l'engagement, y est enraciné. Cela signifie que le regard de l'autre ne provoque pas une inscription du pour-soi dans le monde, mais qu'il objective cette inscription, ce que Sartre nomme transcendance-transcendée, qui consiste dans l'aliénation des structures du pour-soi. Le regard de l'autre fournit donc un caractère objectif au mouvement de transcendance du pour-soi de sorte que ses « structures pour-soi » prennent une forme dégradée: l'engagement devient enracinement (un «engagement-objet $\aleph^{37}$ ), les possibilités deviennent probabilités, etc. "Exister-au-milieu-du-monde-en-présencedes-autres ", dit Sartre, consiste en un approfondissement de l'engagement de fait du pour-soi provoqué par la "visibilité » de cette inscription originelle. L'être regardé est ainsi une caractéristique issue du regard de l'autre, tant que ce pour-soi est toujours engagé au sein du monde. L'autre est la " condition concrète et transcendante $~_{38}$ de l'objectivité du pour-soi et non de sa facticité. En négligeant la structure factice de l'engagement du pour-soi, Merleau-Ponty confondait facticité et objectivité, faisant du pour-soi un pur néant qui ne trouve pas d'adhésion au monde. En ce qui concerne le corps, par exemple, Sartre précise que sa dimension objective ne doit pas être confondue avec sa dimension factice, même si l'une dépend de l'autre: «le corps d'autrui ne doit pas être confondu avec son objectivité. L'objectivité d'autrui est sa transcendance comme transcendée. Le corps est la facticité de cette transcendance. Mais corporéité et objectivité d'autrui sont rigoureusement inséparables ${ }^{39}$. Barbaras, quant à lui, tout en soulignant des aspects négligés par Merleau-Ponty, n'explore pas 
les conséquences de la facticité du pour-soi. Cependant, en montrant les incohérences sartriennes présentes dans ses conditions de base, pointant la chair et l'affectivité originelle comme un moyen de contester le dualisme et de "brouiller " la sphère consciente, il joue Sartre contre Sartre, ouvrant de nouvelles pistes aux investigations de sa cette philosophie.

\section{La question du désir}

On voit bien que le problème du dualisme prend des tournures différentes dans la critique de Barbaras à l'égard de Sartre. Lorsqu'il s'agit de la question du désir, ce point de base de la critique continue à fonctionner, même si ici la différence apparaît au sein d'une certaine proximité. On se risque à signaler cette proximité du fait qu'ici le mouvement de l'auteur se caractérise par une critique de la conception sartrienne du désir, qui se formule simultanément à la reconnaissance d'un "pas décisif » fait par Sartre : celui de mettre le désir sur le même plan que le mouvement intentionnel de la conscience ${ }^{40}$. L'article "Désir et manque dans L'Être et le Néant: le désir manqué » se concentre sur ce thème, dans une ligne annoncée dans l'« Introduction » de Sartre : désir et liberté par Barbaras sur la reprise de la pensée sartrienne à l'intérieur du mouvement phénoménologique actuel en France. En ce sens, comme nous l'avons brièvement mentionné plus haut, Barbaras s'interroge sur le statut phénoménologique de la philosophie sartrienne dans la mesure où elle propose un être transphénoménal sur le mode de l'en-soi. Cependant, les conséquences sont maintenant explorées par le biais du désir afin de montrer combien cette base nuit à l'avancée de Sartre dans l'équivalence qu'il établit entre désir et ouverture intentionnelle, c'est-à-dire qu'il s'agit d'un problème qui concerne le «fondement de l'ouverture du pour-soi à l'en-soi, du mouvement intentionnel $»^{41}$. Car la «version sartrienne de la corrélation $»^{42}$, qui consiste dans le surgissement de la conscience préréflexive par une différenciation avec l'en-soi, apporte nécessairement comme conséquence la conception du désir comme manque. Pour reprendre les mots de Barbaras :

Le désir désigne bien une relation constitutive et non thématique à l'objet, telle que la transcendance de cet objet se trouve préservée dans cette relation même et, en cela, il délivre un sens du sujet conforme à l'a priori universel de la corrélation. Cependant, nous voudrions montrer qu'en caractérisant le désir comme manque, Sartre en manque justement l'essence et s'interdit de penser la corrélation au moment même où il semblait en mesure de le faire ${ }^{43}$.

Bref, l'avancée que représente l'approche de l'intentionnalité en termes de désir est aussitôt gravement compromise par une caractérisation du désir comme manque d'un certain genre, où l'essence du désir, et avec elle la possibilité de la corrélation, se trouve purement et simplement dissoute ${ }^{44}$.

16 Autrement dit, en pensant l'en-soi de manière finalement réaliste, Sartre peut concevoir l'autre pôle de la corrélation - le sujet désirant - comme un manque qui doit être comblé par ce qu'il n'est pas : au fond, le sujet est le néant qui cherche à être rempli par l'être qu'il n'est pas et ne peut jamais être. Encore une fois, le néant ne se trouve pas ici dans une simple position de « repos " par rapport à l'être, mais dans le mode d'une relation qui est maintenant manque ; qui le fait surgir comme n'étant pas cet être, soit comme une manière de (ne pas) être : «En tant que manque, la négation n'est que l'envers d'une position, et non son autre : elle est la position différée et non sa suppression " ${ }^{45}$. L'argument se complexifie lorsque Barbaras analyse "l'événement absolu » qu'est l'émergence du pour-soi à partir du « sacrifice » de l'en-soi, mots utilisés 
par Sartre lui-même. Ce faisant, l'auteur est à nouveau attentif à la structure de la facticité du pour-soi (bien qu'il ne le souligne pas en ces termes), mais cela ne lui suffit pas pour conclure qu'il existe une sorte de « communauté ontologique » entre pour-soi et en-soi, qui permettrait de penser une théorie du désir capable d'appréhender phénoménologiquement son essence. En effet, l'article réunit trois formes de relations du pour-soi à l'en-soi, sans toutefois les différencier suffisamment : 1) la relation du pour-soi à l'en-soi qu'il est, qui se réfère à son surgissement en tant qu'« événement absolu » ou néantisation de soi ; 2) la relation du pour-soi à l'en-soi qu'il n'est pas, qui établit les deux faces d'intentionnalité que sont la négation interne et la position d'un en-soi transcendant ; 3) la relation du pour-soi avec la totalité souhaitée en-soi-poursoi, nommée être de la valeur, qui ne peut être caractérisé exactement comme un ensoi. Ces trois relations sont abordées tout au long du texte, bien que jamais explicitées de la sorte. Ceci fait passer Barbaras d'une analyse de "l'acte ontologique», premier point, aux deux autres en vue d'établir un rapport entre eux à partir d'une conception du pour-soi comme néant. C'est-à-dire que l'analyse de l'acte ontologique, qui est celui qui atteste de la structure de la facticité du pour-soi ou de ce qui « reste de l'en-soi dans le pour-soi $»^{46}$, n'apporte pas assez de visibilité à cet en-soi qui est toujours aussi le pour-soi (dans la mesure où l'acte ontologique est une néantisation incessante de ce que l'on est, et n'est pas uniquement présent au moment métaphysique de son apparition), pour que l'on puisse remettre en question les conclusions issues des deux autres relations. Ainsi, l'argument se concentre principalement sur la corrélation sartrienne entre négativité et positivité pures, nuisant à la théorie du désir en considérant ce dernier comme manque à combler :

Il reste à préciser les raisons de cet échec, qui résident bien dans le point de départ lui-même, c'est-à-dire dans les présupposés ontologiques qui commandent le recours à la théorie du manque pour rendre compte du rapport du pour-soi à l'ensoi. Si la corrélation est manquée dans l'analyse du désir, c'est qu'elle l'est dès le commencement. Tout repose sur ce que l'on pourrait nommer la dissymétrie ontologique de la phénoménologie sartrienne, qui manifeste une forme de naïveté, c'est-à-dire de soumission à l'attitude naturelle, compromettant gravement la démarche phénoménologique ${ }^{47}$.

17 À partir de là, nous pouvons dire que si la critique indique en effet une notion problématique de l'en-soi comme pure positivité, il n'en va pas de même pour le néant comme pure négativité, étant donnée la structure de la facticité. Si nous ne pouvons reprendre que certains points soulevés par Barbaras dans ce texte, qui mériterait par ailleurs un travail détaillé à part, nous pouvons nous organiser sur la base des différents types de relations que nous avons énumérées ci-dessus afin d'éclairer une question centrale : la relation du pour-soi manquant avec la valeur concernant le désir. Si Sartre part d'une division qui est en réalité un « abîme » entre le pour-soi et l'en-soi, comment le pour-soi peut-il désirer une totalité qui soit une contradiction ontologique ? Comment peut-il y avoir du désir sans « horizon de réconciliation $»^{48}$ ? La pure positivité de l'en-soi placé au préalable conduit alors à la conception de cette totalité manquante comme une sorte de non-être, puisqu'il s'agit du désir d'une conciliation ontologique rendue impossible par le dualisme de base. Ainsi, la nature de la relation intentionnelle établie par Sartre compromet de la même manière la relation du pour-soi avec la valeur, un point qui est très clairement mis en évidence dans $L e$ désir et le monde :

[...] les théories du désir qui, telle celle de Sartre, reconnaissant une séparation au cœur du désir, la poussent au bout d'elle-même en la comprenant comme 
séparation entre deux sens de l'être aussi opposés que possible, bref entre le néant et l'être. Si le pour-soi est néant, on se demande comment il pourrait désirer ce radicalement autre que lui-même qu'est l'être pour réaliser le soi comme en-soi pour-soi ${ }^{49}$.

Pour Barbaras, seule une "communauté ontologique » entre le désir et le désiré rend possible la compréhension du désir, puisque «le désir d'un impossible est un désir impossible ${ }^{50}$. L'auteur pense cependant qu'il y a bien une séparation entre le désir et le désiré, mais qui ne serait pas une séparation ontologique qui supprimerait toute " appartenance à la même terre » ${ }^{51}$, mais plutôt une séparation qui se fasse "sur fond d'une communauté qu'elle ne menace pas $\|^{52}$. Séparation est alors le terme utilisé par Barbaras comme alternative pour penser le désir en termes de manque, celui-ci restant attaché au paradigme de la division entre le positif et le négatif. Pour cette même raison, il ne s'agit pas non plus d'une conception du désir comme affirmation, qui, selon lui, se trouve liée aux modèles substantialistes qui orientent une certaine manière de poser cette question : «l'alternative n'est pas entre le négatif et le positif, comme si le sujet du désir ne pouvait être que privé ou complet, vide ou plein [...] par-delà le vide et le plein, le manque et le conatus, il y a la séparation ${ }^{53}$. En déterminant le désir comme un manque, Sartre rend alors justice à la conception substantielle de la négativité selon Barbaras, même s'il fait la distinction entre le manque et le besoin, le besoin étant généralement lié à la notion de sujet qui serait « privé » de quelque chose ${ }^{54}$.

Il reste que d'aucuns pourraient dire que cette critique se fait au sein d'une certaine proximité, qui établit aussi sa distance. Un aspect de cette proximité est soulevé, comme nous l'avons déjà mentionné, par Barbaras lui-même, qui célèbre l'identification faite par Sartre entre désir et intentionnalité : le désir comme ouverture même d'un sujet à ce qui n'est pas lui, comme ce qui conditionne l'apparition d'un monde. Nous y ajoutons d'autres aspects : dans la philosophie de Barbaras, même si le désir n'est pas manque, il est « insatiable » en ce qu'il ne concerne pas le fait d'« avoir » quelque chose, mais le désir, ainsi que pour Sartre, est désir d'être, plus spécifiquement, désir d'être soi-même : «[...] le désir est désir de soi, aspiration à être, quête ontologique $\aleph^{55}$. Par ailleurs, l'être désirant, même s'il ne manque de rien, n'est pas plein : il est « caractérisé par une incomplétude fondamentale, par un défaut d'être, et c'est pourquoi rien ne peut le combler $\aleph^{56}$. Le désir est aussi «le mouvement par lequel un sujet tend à s'approprier son être, à se rejoindre lui-même $[. ..] »^{57}$. Le sujet est donc, hors de lui-même, un sujet " exilé ». Ces dernières affirmations nous renvoient à l'affinité présente dans les deux conceptions qui envisagent un sujet incomplet, qui cherche à être lui-même en dehors de lui-même, de sorte que cette recherche est insatiable ; ainsi qu'à la distinction entre désir et besoin et au questionnement du désir posé sur un plan ontologique ${ }^{58}$. Cependant, comme nous le soulignons brièvement, le positionnement dualiste de Sartre ne permettrait pas, aux yeux de Barbaras, de penser comment l'exil propre à la séparation de soi n'est possible que s'il y a appartenance attestée par la communauté ontologique entre des modes qui sont en jeu dans la corrélation phénoménologique. Il reste à savoir, à partir de la reprise de la question de la facticité chez Sartre - la structure qui empêche la conception du pour-soi comme néant pur et qui conteste le dualisme -, quelles seraient alors les implications de la conception du désir comme manque, et en quoi la chair garantirait une expérience effective de l'autre, afin de remettre en cause les affinités et les oppositions entre les deux auteurs. 


\section{NOTES}

1. M. Merleau-Ponty, Le Visible et l'Invisible, p. 120 ; apud. R. Barbaras, De l'être du phénomène. Grenoble, Jérôme Millon, 2001, p. 140.

2. R. Barbaras, De l'être du phénomène. Grenoble, Jérôme Millon, 2001, p. 140.

3. M. Heidegger, Lettre sur l'humanisme. Paris, Gallimard, 2008, p.85, trad. par A. Préau.

4. R. Barbaras, De l'être du phénomène, op. cit., p. 135.

5. R. Barbaras, La Perception. Essai sur le sensible. Paris, Vrin, 2009, p. 71.

6. Husserl fait une distinction entre Real ou Realität, qui correspond à la réalité naturelle, mondaine, et Reel, qui relève de la réalité de la sphère immanente des vécus. Cf. E. Husserl, Idées directrices pour une phénoménologie et une philosophie phénoménologiques pures. Tome premier : Introduction générale à la phénoménologie pure, trad. par P. Ricœur, Paris : Gallimard, 2013, p. 553 (désormais Ideen I).

7. J.-P. Sartre, L'Être et le Néant. Paris, Gallimard, 2012, p. 27-28.

8. R. Barbaras, La Perception, op. cit., p. 52.

9. Ibid., p. 58.

10. J.-P. Sartre, Situations I : Essais critiques. Paris, Gallimard, 1989.

11. R. Barbaras, La Perception, op. cit., p. 64.

12. J.-P. Sartre, L'Être et le Néant, op. cit., p. 27

13. Ibid., p. 28.

14. Ibid., p. 28.

15. Ibid., p. 28, (nous soulignons).

16. R. Barbaras, "Désir et manque dans L'être et le néant : le désir manqué », Sartre: désir et Liberté, R. Barbaras (éd.), Paris, PUF, 2005, p. 139.

17. Ibid., p. 115.

18. Ibid., p. 135.

19. Ibid., p. 136.

20. Barbaras explique que Merleau-Ponty critique plus la «positivité » de l'essence husserlienne que l'essence elle-même, ce qui fait qu'il préserve la dimension de la signification. Alors que Sartre nie l'essence au nom d'une idée de néant qui hérite, en fait, de sa positivité. En d'autres termes, Sartre attaquerait l'essence au lieu de sa positivité, et ce faisant, il rejette la médiation de l'essence en faveur d'un contact immédiat et frontal avec l'être. $C f$. De l'être du phénomène, op. cit., p. 144-145.

21. R. Barbaras, Dynamique de la manifestation, Paris, Vrin, 2013, p. 80.

22. Ibid., p. 77.

23. Nous montrons en détail cette critique de Merleau-Ponty à Sartre, ainsi que ses limites dans un autre article : F. Alt, « Limites da crítica de Merleau-Ponty a Sartre em O Visível e o Invisível », Ekstasis: Revista de Hermenêutica e Fenomenologia 6 (1), 2017, 51-65.

24. R. Barbaras, De l'être du phénomène, op. cit., p. 140-141.

25. R. Barbaras, De l'être du phénomène, op. cit., p. 142.

26. R. Barbaras, De l'être du phénomène, op. cit., p. 159.

27. M. Merleau-Ponty, Le Visible et l'Invisible, op. cit., p. 99.

28. R. Barbaras, De l'être du phénomène, op. cit., p. 160.

29. Nous approfondissons ces points dans le chapitre «L'événement : les spectres de l'en-soi en tant que zones d'opacité » de notre thèse de doctorat L'Hantologie de Sartre: sur la spectralité dans L'Être et le Néant. Sous la direction des professeurs Renaud Barbaras (Paris I) et Marcos Gleizer (UERJ). 
30. R. Barbaras, "Le corps et la chair dans la troisième partie de L'être et le néant ", in J.-M. Mouillie (éd.) Sartre et la phénoménologie, Fontenay-aux-Roses, ENS, p. 280-281.

31. Ibid., p. 281.

32. Cabestan précise que, contrairement à Merleau-Ponty, Sartre ne connaissait pas ce texte, qui était d'ailleurs pendant longtemps inconnu en France. Cependant, c'est justement en opposition à l'approche de la corporéité au travers du phénomène de la double sensation que Sartre réalisera sa description du corps. P. Cabestan, Qui suis-je ?, Paris, Hermann, 2015, p. 158

33. Ibid., p. 282.

34. Ibid., p. 280.

35. Ibid., p. 293.

36. Ibid., p. 292.

37. J.-P. Sartre, L'Être et le Néant, p. 331.

38. Ibid., p. 313.

39. Ibid., p. 391.

40. R. Barbaras, "Désir et manque dans L'être et le néant: le désir manqué. » in Sartre: désir et liberté, op. cit., p. 115.

41. Ibid., p. 128.

42. Ibid., p. 118.

43. Ibid., p. 115.

44. Ibid., p. 125.

45. Ibid., p. 123.

46. J.-P. Sartre, L'Être et le Néant, op. cit., p. 120.

47. Ibid., p. 135.

48. R. Barbaras, « Désir et manque dans L'être et le néant: le désir manqué. », op. cit., p. 132.

49. R. Barbaras, Le Désir et le monde. Paris, Hermann, 2016, p. 121.

50. Idem.

51. Idem.

52. Ibid., p. 120.

53. Ibid., p. 119.

54. Cf. Ibid., p. $119 ; 2005$, p. $127 ;$ p. 132, p. 134

55. Ibid., p. 117.

56. Ibid., p. 118.

57. Idem.

58. Bien que Barbaras passe d'une phénoménologie à une ontologie tandis que Sartre fait l'inverse. 